\title{
PERFECT TENSORS ON A MANIFOLD
}

\author{
GERALD D. LUDDEN
}

\section{Introduction}

In one of a series of papers on the holonomy group, Hlavaty [2] calls the infinitesimal holonomy group of a connection perfect if its Lie algebra can be found from the curvature tensor alone, that is, the covariant derivatives of the curvature tensor will add nothing new to this Lie algebra. In [4] we generalized this definition to an arbitrary tensor and gave a necessary and sufficient condition that a tensor be perfect. This condition was expressed in terms of restrictions on a certain set of local tensor fields. In this paper, we call a set of tensor fields perfect if they satisfy this condition in every coordinate neighborhood.

Our perfect set of tensor fields reduces to several well-known concepts in special cases. For example, if there is only one tensor field in a perfect set, this tensor field is recurrent (or covariant constant). Also, if the tensor fields in a perfect set are all vector fields, then their linear span at all points of the manifold form a parallel field of planes (see Walker [5]).

To a given tensor field on a manifold $M$, Chern [1] has associated a set of functions on the bundle of frames over $M$. Wong [7] has given a necessary and sufficient condition on these functions such that the associated tensor is covariant constant or recurrent. He also gives [8] a necessary and sufficient condition on these functions so that there exists a connection on the manifold $M$, with respect to which the associated tensor is covariant constant or recurrent. The present paper extends Wong's results to a perfect set of tensors. In this regard, Wong's theorems [7, Theorem 3.9], [8, Theorem 1.2] are special cases of Theorems 2.4 and 2.8. Using our characterization of perfect tensor fields, in $\$ 5$ we are able to prove a fundamental result on fields of planes, namely, every field of planes on $M$ is parallel with respect to some connection on $M$.

In $\S 3$, we examine the set of covectors that occur in the definition of a perfect set of tensors. A necessary and sufficient condition is obtained guaranteeing that the recurrence covector of a recurrent tensor is locally a gradient. This is then generalized to the set of covectors mentioned above. $\$ 4$ is devoted to applications of the previous results.

This paper is condensation of a part of the author's doctoral dissertation presented to the University of Notre Dame. The author would like to express

Received July 24, 1967, and, in revised form, November 20, 1967. 
his sincere thanks to his research director Dr. Cecil B. Mast for his help and encouragement.

\section{Preliminaries}

In this section we will summarize some well-known results on linear connections. The proofs of the various theorems can be found in Chern [1] and Wong [7]. Here also, we will fix our notation.

Let $M$ be a connected, $C^{\infty}$ manifold of dimension $n$, and let $B$ denote the total space of the frame bundle over $M$. Next let $B(M, G)$ denote the principal fiber bundle over $M$ with group $G$, where $G$ is the general linear group and $\pi$ the natural projection of $B$ onto $M$. If $x \in M$, then $z(x) \in B$ will denote a frame at $x$ and thus, $\pi(z(x))=x$.

Let $\left(U, x^{i}\right)$ be a coordinate neighborhood in $M$. If $z(x)$ is a frame at $x \in U$, the tangent vectors $X_{\alpha}(x)(\alpha=1, \cdots, n)$ of $z(x)$ can be expressed as $X_{\alpha}(x)=$ $u_{\alpha}^{i}\left(\partial / \partial x^{i}\right)_{x}$ (note that the summation convention is used throughout this paper), where $u_{\alpha}^{i}$ are $n^{2}$ real numbers such that det $u_{\alpha}^{i} \neq 0$. The following theorem will be stated for a tensor of type $(2,1)$, although it is also true for tensors of arbitrary type.

Theorem 1.1. To any tensor $W$ of type $(2,1)$ on $M$, there corresponds a set of $n^{3}$ functions $\tilde{W}_{\gamma}^{\alpha \beta}$ on $B$ such that for any $z \in B$ and any $g \in G$ we have

$$
\tilde{W}_{\gamma^{\prime}}^{\alpha^{\prime} \beta^{\prime}}(z g)=\tilde{W}_{\gamma}^{\alpha \beta}(z) g_{\gamma}^{-1 \alpha^{\prime}} g_{\beta}^{-1 \beta_{\beta}^{\prime}} g_{\tau^{\prime}}^{\gamma},
$$

where $g_{\alpha}^{-1 \alpha^{\prime}}$ is the inverse matrix of $g_{\alpha^{\prime}}^{\alpha}$. Conversely, to any such $n^{3}$ functions on $B$ satisfying (1.2) there corresponds a tensor of type $(2,1)$ on $M$. Moreover, if $\left(U, x^{i}\right)$ and $\left(\pi^{-1}(U),\left(x^{i}, u_{\alpha}^{i}\right)\right)$ are local coordinate systems in $M$ and $B$ respectively, then

$$
\tilde{W}_{\tau}^{\alpha \beta}(z)=W_{k}^{i j}(x) u_{i}^{\alpha} u_{j}^{\beta} u_{\tau}^{k}
$$

where $z=\left(x^{i}, u_{\alpha}^{i}\right)$.

We will write (1.2) as

$$
\tilde{W}(z g)=g^{-1} \tilde{W}(z)
$$

The quantities $\tilde{W}_{r}^{\alpha \beta}(z)$ are usually called the non-holonomic components of tensor $W_{k}^{i j}$.

Let $\gamma=\left(\gamma_{j}^{i}\right)$ be the matrix of one-forms given by a connection on $M$ in a coordinate system $\left(U, x^{i}\right)$. Then (using an obvious notation) the $n$ one-forms $d x \cdot u^{-1}$ and the $n^{2}$ one-forms $d u \cdot u^{-1}+u \cdot \gamma \cdot u^{-1}$, defined in each $\pi^{-1}(U)$, piece together to form $n$ one-forms $\theta=\left(\theta^{\alpha}\right)$ and $n^{2}$ one-forms $\omega=\left(\omega_{\nu}^{\lambda}\right)$ respectively, globally defined on $B$. A vector tangent to $B$ is said to be horizontal if it annihilates all of the $n^{2}$ one-forms $\omega_{\nu}^{2}$, and a sectionally smooth curve in $B$ is 
called horizontal if its tangent vectors are all horizontal where defined. A horizontal curve $z(t)$ in $B$ is called a lift of a curve $x(t)$ in $M$ if $\pi(z(t))=x(t)$ for each $t$. For any $z_{0} \in B$, let $B\left[z_{0}\right]$ be the set of all points in $B$ that can be joined to $z_{0}$ by a sectionally smooth horizontal curve. We quote the following lemmas.

Lemma 1.4. Any two points in $M$ can be joined by a sectionally smooth curve.

Lemma 1.5. Given any sectionally smooth curve $x(t), 0 \leq t \leq 1$, in $M$ and any $z_{0} \in \pi^{-1}(x(0))$, then there exists a unique lift $z(t)$ of $x(t)$ such that $z(0)=z_{0}$.

Lemma 1.6. For any $x \in M$ and any $z_{0} \in B, \pi^{-1}(x) \cap \mathrm{B}\left[z_{0}\right]$ is non-empty.

Lemma 1.7. For any two points, $z_{0}$ and $z_{1}$, of $B$, there exists a $g \in G$ such that $B\left[z_{1}\right]=B\left[z_{0} g\right]=\left\{z^{\prime} \mid z^{\prime}=z g\right.$ for some $\left.z \in B\left[z_{0}\right]\right\}$.

Lemm 1.8. If $\tilde{W}_{T}^{\alpha \beta}(z)$ are the $n^{3}$ functions on $B$ corresponding to the tensor $W_{i}^{i j}$ on $M$, then the absolute differentials $h d \tilde{W}_{\gamma}^{\alpha \beta}$ (i.e. the horizontal part of $\left.d \tilde{W}_{\tau}^{\alpha \beta}\right)$ are related to the covariant differential $\delta W_{k}^{i j}$ by

$$
h d \tilde{W}_{\tau}^{\alpha \beta}(z)=\delta W_{k}^{i j} \cdot u_{i}^{\alpha} u_{j}^{\beta} u_{\gamma}^{k} .
$$

Furthermore, the $n^{4}$ functions $D_{c} \tilde{W}_{r}^{\alpha \beta}$ on $B$, defined by $h d \tilde{W}_{r}^{\alpha \beta}=D_{\varepsilon} \tilde{W}_{\gamma}^{\alpha \beta} \theta^{c}$, correspond to $\nabla_{m} \mathrm{~W}_{k}^{i j}$ in a similar fashion.

It is known that any principal fiber bundle which satisfies the second axiom of countability admits a connection. The following theorem will enable us to extend a connection which we put on a closed subbundle to all of $B(M, G)$.

Theorem 1.10. Let $f: B^{\prime}\left(M^{\prime}, G^{\prime}\right) \rightarrow B(M, G)$ be a homomorphism of principal fiber bundles with corresponding homomorphism $f: G^{\prime} \rightarrow G$ such that the induced mapping $f: M^{\prime} \rightarrow M$ is a diffeomorphism of $M^{\prime}$ onto $M$. Let $\Gamma^{\prime}$ be a connection in $B^{\prime}$. Then there is a unique connection in $B$ such that the horizontal subspaces of $\Gamma^{\prime}$ are mapped by $f$ into horizontal subspaces of $\Gamma$.

\section{Perfect sets of tensor fields}

In this section, we will define the concept of perfect sets of tensor fields and obtain a characterization of them. The theorems which provide such characterization are a generalization of similar theorems of Wong [7] that characterize a recurrent tensor field.

Definition 2.1. A set $\left\{W_{A} \mid A=1, \cdots, r\right\}$ of tensor fields of the same type on $M$ is perfect if

(i) the $W_{A}$ 's are linearly independent at at least one point of $M$ (i.e. there is $x \in M$ such that $C^{A} W_{A}(x)=0$ implies $C^{A}=0$ for all $A$, where $C^{A}$ are real numbers), and

(ii) in any coordinate neighborhood we have $\nabla_{h} W_{A}=F_{A h}^{B} W_{B}$, for some set of local covariant vector fields $F_{A h}^{B}$.

In proving the following theorems we frequently specify the $W_{A}$ 's to be of a particular type (usually of type $(1,1)$ ) for convenience. However, the type 
is completely arbitrary. We might mention here that if $r=1$, then the perfect set of tensors is just a recurrent tensor (or covariant constant tensor if $F_{1 h}^{1}=$ 0 ), or if the vectors $F_{A h}^{B}$ are of the form $\delta_{A}^{B} V_{A h}$ (no sum on $A$ ) on $M$, then the perfect set of tensors is just a set of recurrent (or covariant constant) tensors. Also, if the tensors $W_{A}$ are all of type $(1,0)$ (i.e. vectors) then the set $s p\left\{W_{A}\right\}$ of all linear combinations of the $W_{A}(x)$ 's at each point $x$ of $M$ forms a parallel field of planes on $M$ (see Walker [5]).

Theorem 2.2. If $\left\{W_{A} \mid A=1, \cdots, r\right\}$ is a perfect set of tensors, then along any sectionally smooth horizontal curve in $B$ there exists a matrix function $\phi_{A}^{B}(t)$ such that

$$
\tilde{W}_{A}(t)=\phi_{A}^{B}(t) \tilde{W}_{B}(0),
$$

where $\tilde{W}_{A}$ represents the set of functions on $B$ corresponding, in the sense of Theorem 1.1, to the tensor $W_{A}$. Also det $\phi_{A}^{B}(t)$ does not vanish.

Proof. Suppose the horizontal curve $z(t), 0 \leq t \leq 1$, lies in a coordinate neighborhood $\pi^{-1}(U)$ so that $x(t)=\pi(z(t))$ is a smooth curve in $M$. Assume the $W_{A}$ 's are of type $(1,1)$ on $M$. Now $\frac{d}{d t}\left(\tilde{W}_{A \beta}^{\alpha}\right)=\left\langle\frac{d z}{d t}, d \tilde{W}_{A \beta}^{\alpha}\right\rangle$, where $\langle$,$\rangle denotes the action of the form on the vector. By Lemma 1.8, since z(t)$ is horizontal, we then have

$$
\begin{aligned}
& \frac{d}{d t}\left(\tilde{W}_{A \beta}^{\alpha}\right)=\left\langle\frac{d z}{d t},\left(\delta W_{A j}^{i}\right) u_{i}^{\alpha} u_{\beta}^{j}\right\rangle=\left\langle\frac{d x}{d t}, \delta W_{A j}^{i}\right\rangle u_{i}^{\alpha} u_{\beta}^{j} \\
& \quad=\left(\frac{d x^{k}}{d t} \cdot \nabla_{k} W_{A j}^{i}\right) u_{i}^{\alpha} u_{\beta}^{j}=\left(\frac{d x^{k}}{d t} F_{A k}^{B} W_{B j}^{i}\right) u_{i}^{\alpha} u_{\beta}^{j}=f_{A}^{B}(t) \tilde{W}_{B \beta}^{\alpha},
\end{aligned}
$$

where $f_{A}^{B}(t)=\frac{d x^{k}}{d t} F_{A k}^{B}$. Therefore, $W_{A \beta}^{\alpha}(1 \leq \alpha, \beta \leq n)$ are $n^{2}$ solutions of the system of differential equations

$$
\frac{d \phi_{A}}{d t}=f_{A}^{D}(t) \phi_{D}
$$

Let $\phi_{A}^{B}(t)(1 \leq B \leq r)$ be $r$ linearly independent solutions of this system of differential equations such that $\phi_{A}^{B}(0)=$ identity matrix. Then

$$
\tilde{W}_{A \beta}^{\alpha}(t)=\tilde{W}_{B \beta}^{\alpha}(0) \phi_{A}^{B}(t) \text {. }
$$

Moreover, since

$$
\operatorname{det} \phi_{A}^{B}(t)=\operatorname{det} \phi_{A}^{B}(0) \exp \left(\int_{0}^{t} \operatorname{trace} f_{A}^{B}(t) d t\right)
$$


(see Hochstadt $[3, \S 2.8]$ ) and $f_{A}^{B}(t)=\frac{d x^{k}}{d t} F_{A k}^{B}$ are $C^{\infty}$ functions, we have that $\operatorname{det} \phi_{A}^{B}(t)$ is nowhere zero. This finishes the proof of the theorem.

By definition, the $W_{A}$ 's are linearly independent at at least one point of $M$, say at $x_{0}$. Let $z_{0} \in \pi^{-1}\left(x_{0}\right)$ and let $x_{1}$ be any point in $M$. Since $\pi^{-1}\left(x_{1}\right) \cap B\left[z_{0}\right]$ is non-empty, there is a sectionally smooth horizontal curve $z(t)$ in $B$ such that $z(0)=z_{0}$ and $\pi(z(1))=x_{1}$. Thus $\tilde{W}_{A}(z(1))=\phi_{A}^{B}(1) \tilde{W}_{B}\left(z_{0}\right)$, where $\operatorname{det} \phi_{A}^{B}(1) \neq 0$. This proves

Corollary 2.3. If $\left\{W_{A} ! A=1, \cdots, r\right\}$ is a perfect set of tensors on $M$, the $W_{A}$ 's are linearly independent at every point of $M$.

We are now in a position to prove the main theorem that characterizes a perfect set of tensors :

Theorem 2.4. The set of tensors $\left\{W_{A} \mid A=1, \cdots, r\right\}$ is perfect if and only if the $\tilde{W}_{A}$ 's, with domain restricted to any $B\left[z_{0}\right]$, are linearly independent on $B\left[z_{0}\right]$ and given by

$$
\tilde{W}_{A}(z)=\phi_{A}^{B}(z) C_{B}, \quad z \in B\left[z_{0}\right],
$$

where det $\phi_{A}^{B}(z)$ is nowhere zero on $B\left[z_{0}\right]$ and the $C_{B}$ 's are constants.

Proof. We should note that if $\tilde{W}_{B}$ stands for $\tilde{W}_{B \beta}^{\alpha \cdots *}$, then $C_{B}$ stands for $C_{B \beta} a \ldots$.... Let us again suppose the $W_{A}$ 's are tensors of type $(1,1)$. Since any $z$ in $B\left[z_{0}\right]$ can be joined to $z_{0}$ by a sectionally smooth horizontal curve in $B$, the "only if" part of the theorem follows immediately from Theorem 2.2.

To prove the "if" part of the theorem, take any $x_{1} \in M$ and any coordinate neighborhood $U$ of $x_{1}$. Then there is a $z_{1} \in B\left[z_{0}\right]$ such that $\pi\left(z_{1}\right)=x_{1}$. Let $x(t), 0 \leq t \leq 1$, be any smooth curve in $U$ with $x\left(t_{1}\right)=x_{1}$, where $0 \leq t_{1} \leq 1$, and let $z(t)$ be the lift of $x(t)$ such that $z\left(t_{1}\right)=z_{1}$. Therefore $z(t)$ is a curve in $\pi^{-1}(U) \cap B\left[z_{0}\right]$, and thus on $z(t)$ we have

$$
\begin{array}{r}
\left(\nabla_{k} W_{A j}^{i}\right) \frac{d x^{k}}{d t} u_{i}^{\alpha} u_{\beta}^{j}=\frac{d}{d t}\left(\tilde{W}_{A \beta}^{\alpha}\right)=\frac{d}{d t}\left(\phi_{A}^{B}\right) C_{B \beta}^{\alpha} \\
=\left(\frac{d}{d t} \phi_{A}^{B} \cdot \phi^{-1 D}\right) \phi_{D}^{C} C_{C \beta}^{\alpha}=f_{A}^{B}(t)\left(W_{B j}^{i} u_{i}^{\alpha} u_{B}^{j}\right),
\end{array}
$$

where $\phi_{B}^{-1 D}$ is the inverse matrix of $\phi_{D}^{B}$, and we have denoted $\frac{d}{d t} \phi_{A}^{B} \cdot \phi^{-1 D}$ by $f_{A}^{D}(t)$. Thus, along the curve $x(t)$, we have $\left(\nabla_{k} W_{A j}^{i}\right) \frac{d x^{k}}{d t}=f_{A}^{B}(t) W_{B j}^{i}$. However, since this is to hold for any smooth curve $x(t)$ with $x\left(t_{1}\right)=x_{1}$, it is a simple matter to show that $\nabla_{k} W_{A j}^{i}=F_{A k}^{B} W_{B j}^{i}$ on $U$ for some set of vectors $F_{A k}^{B}$. This finishes the proof of the theorem.

The preceding theorem demanded that $\tilde{W}_{A}(z)$ be of the form $\phi_{A}^{B}(z) C_{B}$ on any $B\left[z_{0}\right]$. However, if $\tilde{W}_{A}(z)=\phi_{A}^{B}(z) C_{B}$ on $B\left[z_{0}\right]$, then, since $B\left[z_{1}\right]=B\left[z_{0} g\right]$ for some $g \in G$, we have for $z^{\prime} \in B\left[z_{1}\right]$ that 


$$
\tilde{W}_{A}\left(z^{\prime}\right)=\tilde{W}(z g)=g^{-1} \tilde{W}_{A}(z)=g^{-1} \phi_{A}^{B}(z) C_{B}=\phi_{A}^{\prime B}\left(z^{\prime}\right) C_{B}^{\prime},
$$

where $\phi_{A}^{\prime B}\left(z^{\prime}\right)=\phi_{A}^{B}(z)$ if $z^{\prime}=z g$ and $C_{B}^{\prime}=g^{-1} C_{B}$. As a consequence, the $W_{A}$ 's assume the same form on $B\left[z_{1}\right]$ as on $B\left[z_{0}\right]$.

If each of the tensors $W_{A}$ appearing in the statement of Theorem 2.2 is covariant constant (a special case of $\left\{W_{A} \mid A=1, \cdots, r,\right\}$ being perfect), the systems of differential equations which appear in the proof of Theorem 2.2 become homogeneous systems. Hence, the matrix $\phi_{A}^{B}(t)$ obtained from the auxiliary system of equations is a constant matrix. This shows that Corollary 2.7 below (which is Theorem 3.15 in Wong [7]) is immediate. Corollary 2.6 below which is Theorem 3.9 in Wong [7]) is just Theorem 2.4 for the special case where $r=1$.

Corollary 2.6. The tensor $W$ on $M$ is recurrent if and only if the corresponding function $\tilde{W}$ on $B$ have no common zero and are proportional to a set of constants on ony $B\left[z_{0}\right]$.

Corollary 2.7. The tensor $W$ on $M$ is covariant constant if and only if the corresponding functions have no common zero and are constant on any $B\left[z_{0}\right]$.

If we are given a set of tensors on $M$, we might ask if there is any connection on $M$ with respect to which the set is perfect. The answer is given in

Theorem 2.8. Let $\left\{W_{A} \mid A=1, \cdots, r\right\}$ be a given set of tensors on $M$. There exists a connection on $M$ such that this set is perfect with respect to this connection if and only if we can assign to each $x \in M$ a frame $z(x)$ such that the $\tilde{W}_{A}(z(x))$ are linearly independent for each $x \in M$, and $\tilde{W}_{A}(z(x))$ $=\phi_{A}^{B}(z(x)) C_{B}$ for some set of constants $C_{B}$ and some matrix function $\phi_{A}^{B}(z(x))$ on $M$ whose determinant is nonvanishing on $M$.

Proof. Since $\pi^{-1}(x) \cap B\left[z_{0}\right]$ is non-empty for each $x \in M$, Theorem 2.4 proves the "only if" part of the theorem.

Now assume for each $x \in M$ there is a frame $z(x)$ such that $\tilde{W}_{A}(z(x))=$ $\phi_{A}^{B}(z(x)) C_{B}$ for some set of lineary independent constants $C_{B}$ and some matrix function $\phi_{A}^{B}(z(\mathrm{x}))$ on $M$ whose determinant is non-vanishing on $M$.

Define $H$ to be the subgroup of $G$ consisting of all elements $h$ in $G$ for which there exists a non-singular $r \times r$ matrix $\phi_{B}^{A}(h)$ such that $h^{-1} C_{A}=$ $\phi_{A}^{B}(h) C_{B}$. Then $H$ is a non-empty closed subgroup of $G$, and therefore $H$ is a Lie group. In a like manner, define $B_{H}$ to be the set of all points $z$ in $B$ for which there exists a non-singular $r \times r$ matrix $\phi_{A}^{B}(z)$ such that $W_{A}(z)=$ $\phi_{A}^{B}(z) C_{B}$. It is now a simple matter to show that for each $x \in M, B_{H} \cap \pi^{-1}(x)$ $=z(x) H=\{z(x) h \mid h \in \mathrm{H}\}$. Hence, $B_{z}$ is invariant and has no fixed points under the action of $H$. We now will show that $B_{H}$ is a closed submanifold of $B$. Let $z_{n}$ be a sequence of points in $B_{H}$ converging to $z$ in $B$ and let $\tilde{W}_{A}\left(z_{n}\right)$ $=\phi_{A}^{B}\left(z_{n}\right) C_{B}$. Since the functions $\tilde{W}_{A}$ are $C^{\infty}$ on $B$, we have that $\lim _{n \rightarrow \infty}^{\lim } \phi_{A}^{B}\left(z_{n}\right) C_{B}$ $=\tilde{W}_{A}(z)$. The constants $C_{A}$ are linearly independent, so there is a matrix $C^{A}$ such that $C_{B} C^{A}=\delta_{B}^{A}$ (i.e. if $C_{A}$ stands for $C_{A}^{\alpha}$ then $C^{A}$ stands for $C_{\alpha}^{A}$ and $\left.C_{B}^{\alpha} C_{\alpha}^{A}=\delta_{B}^{A}\right)$. Thus, $\lim _{n \rightarrow \infty} \phi_{A}^{B}\left(z_{n}\right)=\tilde{W}_{A}(z) C^{B}=\phi_{A}^{B}(z)$. Substituting this last 
equation in the previous one, we have $\tilde{W}_{A}(z)=\phi_{A}^{B}(z) C_{B}$, which shows that $z \in B_{H}$.

From the above, we see that $B_{H}(M, H)$ is a principal fiber bundle, which is a subbundle of $B(M, G)$. It is well-known that there exists a connection on $B_{H}(M, H)$ which, by Theorem 1.10 , can be extended to $B(M, G)$. Also Theorem 1.10 can be used to show that $B_{H}\left[z_{0}\right]=B\left[z_{0}\right]$ for any $z_{0}$. Since the functions $\tilde{W}_{A}$ satisfy the hypothesis of Theorem 2.4 on all of $B_{H}$, therefore on $B_{H}\left[z_{0}\right]=B\left[z_{0}\right]$, we have that the set $\left\{W_{A} \mid A=1, \cdots, r\right\}$ is perfect with respect to this connection. Hence the proof is finished.

\section{The vectors $F_{A k}^{B}$}

In the case of a recurrent tensor field it is of some interest to determine whether the recurrence covector is locally a gradient. We could also ask this question of the set of vectors $F_{\boldsymbol{A} k}^{B}$. The following theorem gives a necessary and sufficient condition for the vectors $F_{A k}^{B}$ to assume a form resembling a gradient.

Theorem 3.1. Let $\left\{W_{A} \mid A=1, \cdots, r\right\}$ be a perfect set of tensors, so that $\nabla_{k} W_{A}=F_{A k}^{B} W_{B}$ on ony coordinate neighborhood $U$, and for some $z_{0} \in B$ let $W_{A}(z)=\phi_{A}^{B}(z) C_{B}$ for $z \in B\left[z_{0}\right]$. Then

$$
F_{A k}^{B}=-\psi_{C}^{-1 B} \partial_{k} \psi_{A}^{C}
$$

for some matrix function $\psi_{B}^{A}$ on $U$ (here $\psi_{c}^{-1 B}$ is the inverse matrix of $\phi_{c}^{B}$ ) if and only if for each $x \in M, \phi_{A}^{B}(z)$ is constant on $\pi^{-1}(x) \cap B\left[z_{0}\right]$.

Proof. Suppose $\phi_{A}^{B}(z)$ is constant on $\pi^{-1}(x) \cap B\left[z_{0}\right]$ for all $x \in U$, where $U$ is any coordinate neighborhood.

Define a matrix function $\psi_{A}^{B}(x)$ on $U$ by

$$
\phi_{A}^{B}(x)=\phi_{A}^{-1 B}(z),
$$

where $z$ is any point of $\pi^{-1}(x) \cap B\left[z_{0}\right]$. We should note here that if $\tilde{W}_{A}(z)=$ $\phi_{A}^{* B}(z) C_{B}^{\prime}$ on $B\left[z_{1}\right]$, then, since $B\left[z_{1}\right]=B\left[z_{0} g\right]$ for some $g \in G$, we have that

$$
\phi_{A}^{* B}(z)=\phi_{A}^{B}\left(z g^{-1}\right)=\phi_{A}^{-1 B}\left(\pi\left(z g^{-1}\right)\right) \text {. }
$$

It is easy to see that $\psi_{A}^{B}(x)$ is a $C^{\infty}$ function on $U$ (local cross-sections of $B\left[z_{0}\right]$ exist). Therefore, let $W_{A}^{*}$ be $r$ tensors on $U$ given by

$$
W_{A}^{*}(x)=\phi_{A}^{B}(x) W_{B}(x) \text {. }
$$

These tensors are linearly independent since $\operatorname{det} \phi_{A}^{B}(x) \neq 0$. Since the $\phi_{A}^{B}(x)$ are scalars on $U, \tilde{\phi}_{A}^{B}(z)=\psi_{A}^{B}(\pi z)$ on $\pi^{-1}(U) \cap B\left[z_{0}\right]$. Therefore, we have

$$
\tilde{W}_{A}^{*}(z)=\tilde{\phi}_{A}^{B}(z) \tilde{W}_{B}(z)=\phi_{A}^{-1 B}(z) \phi_{B}^{C}(z) C_{C}=C_{A}
$$


on $\pi^{-1}(U) \cap B\left[z_{0}\right]$. By Corollary 2.7 , each tensor $W_{A}^{*}$ is covariant constant on $U$. This implies that

$$
0=\nabla_{k} W_{A}^{*}=\partial_{k} \psi_{A}^{B} \cdot W_{B}+\phi_{A}^{B} F_{B k}^{C} W_{C},
$$

and, since the $W_{B}$ 's are linearly independent, we have that $0=\partial_{k} \psi_{A}^{B}+\psi_{A}^{C} F_{C k}^{B}$. Since det $\psi_{A}^{B} \neq 0$, this last equation can be written as $F_{B k}^{A}=-\psi_{B}^{-1 C} \cdot \partial_{k} \psi_{C}^{A}$ on $U$.

Now suppose that $F_{B k}^{A}=-\psi_{B}^{-1 C} \cdot \partial_{k} \psi_{C}^{A}$ on every coordinate neighborhood $U$, for some matrix function $\psi_{B}^{A}$ defined on $U$. Then the $r$ tensors $W_{A}^{*}$ on $U$, defined by $W_{A}^{*}=\psi_{A}^{B} W_{B}$, are covariant constant on $U$. Therefore $\tilde{W}_{A}^{*}(z)=D_{A}$ on $\pi^{-1}(U) \cap B\left[z_{0}\right]$ for any $z_{0}$, where $D_{A}$ is some set of constants. Thus we see that

$$
\tilde{\psi}_{A}^{B}(z) \tilde{W}_{B}(z)=\tilde{\psi}_{A}^{B}(z) \phi_{B}^{C}(z) C_{C}=D_{A}
$$

on $\pi^{-1}(U) \cap B\left[z_{0}\right]$, where $\tilde{\psi}_{A}^{B}(z)=\phi_{A}^{B}(\pi z)$. Since $\tilde{\phi}_{A}^{B}(z)$ is constant on $\pi^{-1}(x) \cap B\left[z_{0}\right]$ for each $x$ in $U$, this last equation shows that $\phi_{A}^{B}(z)$ must also be constant on $\pi^{-1}(x) \cap B\left[z_{0}\right]$ for each $x \in U$. Since this is true for every $U$, the theorem is proved.

Corollary 3.2. If $W$ is a recurrent tensor with recurrence covector $V$, and $\tilde{W}(z)=\rho(z) C$ on $B\left[z_{0}\right]$, then $V_{m}$ is locally a gradient if and only if $\rho(z)$ is constant on $\pi^{-1}(x) \cap B\left[z_{0}\right]$ for each $x \in M$.

Proof. Let $r=1$ in the theorem. Then we have that

$$
V_{k}=-\psi^{-1} \partial_{k} \psi=-\partial_{k} \ln \psi \text { on } U
$$

if and only if $\rho(z)$ is constant on $\pi^{-1}(x) \cap \mathrm{B}\left[z_{0}\right]$ for each $x \in U$.

In the special case of tensors of type $(1,1)$, we have the following sufficient condition that the recurrence covector is locally a gradient. Its proof uses some of the technique used above.

Theorem 3.3. If $W$ is a recurrent tensor of type $(1,1)$ on $M$ such that trW $\neq 0$ on $M$, then the recurrence covector is locally a gradient.

Proof. Let $U$ be any coordinate neighborhood. By Theorem 2.4, for each $x \in U$, there is a frame $z(x)$ such that $\tilde{W}(z(x))=\rho(x) C$, where $\rho(x) \neq 0$ on $U$. Since local cross-sections of $B\left[z_{0}\right]$ exist, we see that $\rho(x)$ can be assumed to be $C^{\infty}$. Let $W^{*}$ be a tensor on $U$ defined by $W^{*}(x)=\frac{1}{\rho(x)} W(x)$. Now, we see that

$$
\tilde{W}^{*}(z(x))=\frac{1}{\rho(x)} \tilde{W}(z(x))=\frac{1}{\rho(x)} \rho(x) C=C .
$$

Therefore, by Theorem 2.4 and Corollary 2.7 , there is a connection on $U$ respect to which $W^{*}$ is covariant constant on $U$. Let the components of this 
connection be $\Gamma_{j k}^{\prime i}=\Gamma_{j k}^{i}+K_{j k}^{i}$, where $\Gamma_{j k}^{i}$ are the components of the original connection. Therefore, we have

$$
\begin{aligned}
0 & =\nabla_{k}^{\prime} W_{j}^{* i}=\nabla_{k} W_{j}^{* i}+K_{k m}^{i} W_{j}^{* m}-K_{k j}^{m} W_{m}^{* i} \\
& =\left(\partial_{k} \frac{1}{\rho(x)}+\frac{1}{\rho(x)} V_{k}\right) W_{j}^{i}+\rho(x)\left(K_{k m}^{i} W_{j}^{m}-K_{k j}^{m} W_{m}^{i}\right),
\end{aligned}
$$

so contracting we obtain that $0=\left(\partial_{k} \frac{1}{\rho(x)}+\frac{1}{\rho(x)} \mathrm{V}_{k}\right) W_{i}^{i}$. Or $V_{k}=$ $-\rho(x) \partial_{k} \frac{1}{\rho(x)}=-\partial_{k} \ln \frac{1}{\rho(x)}$ on $U$, since $W_{i}^{i}$ is assumed to be non-zero.

\section{Applications}

A large part of a recent paper by Wong [8] was devoted to the question of whether the connection on $M$ can be changed so that a tensor that is recurrent with respect to the original connection is covariant constant with respect to the new connection. We will use the following lemma, which appears as Theorem 1.3 in Wong [8], to study this situation in the case of perfect tensors. This lemma is the homogeneous case of Theorem 2.8 .

Lemma 4.1. Let $W_{A}(A=1, \cdots, r)$ be $r$ given tensors on $M$. There exists a connection on $M$, with respect to which each of the $W_{A}$ is covariant constant, if and only if we can assign a frame $z(x)$ to each $x \in M$ such that $\tilde{W}_{A}(z(x))$ are not all zero, and are independent of $x$ for each $A$.

Theorem 4.2. Let $\left\{W_{A} \mid A=1, \cdots, r\right\}$ be a perfect set of tensors on $M$ (with respect to a given connection). Then for every coordinate neighborhood $U$, there exists a connection on $U$ and $r$ linearly independent tensors $W_{A}^{\prime}$ on $U$ such that each of the $W_{A}^{\prime}$ is covariant constant with respect to this new connection and $\mathrm{sp}\left\{W_{A}^{\prime}(x)\right\}=\operatorname{sp}\left\{W_{A}(x)\right\}$ for each $x$ in $U$.

Proof. Let $U$ be any coordinate neighborhood. By Theorem 2.8 for each $x \in U$ there is a frame $z(x)$ such that $\tilde{W}_{A}(z(x))=\phi_{A}^{B}(x) C_{B}$, where $\phi_{A}^{B}(z)$ is $C^{\infty}$ on $U$ and $\operatorname{det} \phi_{A}^{B}(x) \neq 0$ on $U$. Define $r$ tensors $W_{A}^{\prime}$ on $U$ by

$$
W_{A}^{\prime}(x)=\phi_{A}^{-1 B}(x) W_{B}(x),
$$

where $\phi_{A}^{-1 B}(x)$ is the inverse of $\phi_{A}^{B}(x)$. Since the $W_{A}$ are linearly independent, so are the $W_{A}^{\prime}$ and clearly sp $\left\{W_{A}^{\prime}(x)\right\}=\operatorname{sp}\left\{W_{A}(x)\right\}$ at each $x \in U$. Now

$$
\tilde{W}_{A}^{\prime}(z(x))=\tilde{\phi}_{A}^{-1 B}(z(x)) \tilde{W}_{B}(z(x))=\tilde{\phi}_{A}^{-1 B}(x) \phi_{B}^{C}(x) C_{C}=C_{A} .
$$

Thus, by Lemma 4.1, the proof is finished.

In the special case of tensors of type $(1,0)$, we can go a bit further. We will give two proofs of the following theorem. In the first proof, we will only use the fact that a certain set of vector fields is perfect in order to insure that they 
are linearly independent at all points of $M$. Hence, it will apply to any set of linearly independent vectors on $M$.

Theorem 4.3. Let $\left\{W_{A} \mid A=1, \cdots, r\right\}$ be a perfect set of tensors of type $(1,0)$ on $M$. Then there exists a connection on $M$ such that each of the vectors $W_{A}$ is covariant constant with respect to this connection.

Proof 1. Since the $W_{A}^{i}$ 's are linearly independent at each $x \in M$, we can extend $\left\{W_{A}^{i}(x)\right\}$ to a basis $\left\{W_{\alpha}^{i}(x)\right\}$ for $M_{x}$ at each $x \in M$. Then $\left\{W_{\alpha}^{i}(x)\right\}$ is a frame at $x$, so let $\left\{W_{i}^{\alpha}(x)\right\}$ be its dual coframe. With respect to this set of frames, we have

$$
\tilde{W}_{A}^{\alpha}(z(x))=W_{A}^{i}(x) W_{i}^{\alpha}(x)=\delta_{A}^{\alpha} .
$$

Therefore, by Lemma 4.1 , there is a connection on $M$, with respect to which each of the vectors $W_{A}$ is covariant constant.

Proof 2. Let $U$ be any coordinate neighborhood and let $\nabla_{m} W_{A}^{i}=F_{A m}^{B} W_{B}^{i}$ on $U$. Extend the $W_{A}^{i}$ to a basis $W_{\alpha}^{i}$ of the tangent space $M_{x}$ at each point of $U$, and let $W_{i}^{\alpha}$ denote the corresponding dual bases. This process can be done so that the $W_{i}^{\alpha}$ are $C^{\infty}$ on $U$. Define a tensor $K_{k j}^{i}$ on $U$ by

$$
K_{k j}^{i}=-W_{A}^{i} F_{B k}^{A} W_{j}^{B} .
$$

The $W_{\alpha}^{i}$ and $W_{i}^{\beta}$ can be picked so that $K_{k j}^{i}$ are the components of a $C^{\infty}$ tensor on $M$. Let a new connection $M$ be given whose components on $U$ are

$$
\Gamma_{k j}^{\prime i}=\Gamma_{k j}^{i}+K_{k j}^{i}
$$

where the $\Gamma_{k j}^{i}$ are the components of the original connection. Then

$$
\nabla_{k}^{\prime} W_{A}^{i}=\nabla_{k} W_{A}^{i}+K_{k m}^{i} W_{A}^{m}=F_{A k}^{B} W_{B}^{i}-W_{B}^{i} F_{C k}^{B} W_{m}^{C} W_{A}^{m}=0 .
$$

Thus, the proof is finished.

It is well known (see Wong [7]) that the Lie algebra of the holonomy group at any point $z_{0}$ of $B$ is spanned by the elements $\tilde{R}_{\nu(\alpha \beta)}^{2}(z), 1 \leq \alpha, \beta \leq n$, as $z$ runs through $B\left[z_{0}\right]$. We close this section with the following application of Theorem 2.4 to the holonomy group on $B$.

Theorem 4.4. Let $R$ be the curvature tensor of the given connection on $M$ and suppose $R$ can be globally decomposed as $R=W_{A} \otimes M^{A}(A=$ $1, \cdots, r)$, where the $W_{A}$ 's form a perfect set of tensors of type $(1,1)$ on $M$, and the tensors $M^{-4}$ of type $(0,2)$ are linearly independent on $M$. Then the dimension of the holonomy group is constant on $B$ and is less than or equal to $n(n-1) / 2$.

Proof. Since the $M^{A}$ 's are linearly independent tensors that are skewsymmetric at each point of $M$, we see that $r \leq n(n-1) / 2$. Now, we have $\tilde{W}_{A \beta}^{\alpha}(z)=\phi_{A}^{B}(z) C_{B \beta}^{\alpha}$ on any $B\left[\mathrm{z}_{0}\right]$, where $\phi_{A}^{B}(z)$ is a non-singular matrix function defined on $B\left[z_{0}\right]$. Therefore, $R_{\nu(\alpha \beta)}^{\lambda}(z)=C_{B \nu}^{\lambda} \phi_{A}^{B}(z) \bar{M}_{(\alpha \beta)}^{A}(z)$ on $B\left[z_{0}\right]$ and thus, 
the holonomy algebra at $z_{0}$ is spanned by linear combinations of the $r$ matrices $C_{A}$. Since the $\bar{M}_{\alpha B}^{A}\left(z_{0}\right)$ are linearly independent, the $r \times n^{2}$ matrix $\left(\bar{M}_{\alpha \beta}^{A}\left(z_{0}\right)\right)$, where $A$ represents the row and the pair $\alpha \beta$ represents the column, has rank $r$. Hence, this matrix has $r$ linearly independent columns say $\bar{M}_{\bar{A}}^{A}\left(z_{0}\right)(\bar{A}=$ $1, \cdots, r)$. Now $\operatorname{det}\left(\phi_{A}^{B}\left(z_{0}\right) \bar{M}_{\bar{A}}^{A}\left(z_{0}\right)\right)=\operatorname{det} \phi_{A}^{B}\left(z_{0}\right) \operatorname{det} \bar{M}_{\bar{A}}^{A}\left(z_{0}\right) \neq 0$. Therefore, the $r$ linearly independent matrices $C_{B_{\nu}}^{2} \phi_{A}^{B}\left(z_{0}\right) \bar{M}_{A}^{A}\left(z_{0}\right)$ are all in the holonomy algebra at $z_{0}$. Hence, the dimension of the holonomy group at any $z$ in $B$ is $r$.

\section{A generalization}

As mentioned before, if a perfect set of $r$ tensors consists of tensors of type $(1,0)$, then the linear span of these tensors at each point of $M$ produces a parallel field of $r$-planes on $M$. However, if we are given a parallel field of $r$-planes on $M$, in general we cannot find $r$ vectors on $M$ that span the parallel field. This is so, since $r$-distributions do not always exist for arbitrary $r$. We would like to use our results to prove the following well-known theorem on $r$-planes:

Theorem 5.1. If $M$ admits a $C^{\infty}$ field of planes, then $M$ admits a connection with respect to which this field of planes is parallel.

This theorem is in the paper by Willmore [6]. If we can find $r$ vectors globally defined on $M$ that span the field of planes, then applying the method of proof 1 of Theorem 4.3 and using Theorem 2.8, the proof of Theorem 5.1 is immediate. However, to prove the general case we need the following generalization of Theorem 2.8 .

Theorem 5.2. Let there be given $r$ tensors $W_{A}(A=1, \cdots, r)$ on each coordinate neighborhood $U$ such that on $U \cap U^{*}$ we have $W_{A}^{*}=\phi_{A}^{B} W_{B}, W_{B}=$ $\psi_{A}^{B} W_{B}^{*}$, where the $W_{A}$ and $W_{A}^{*}$ are the tensors defined on the neighborhoods $U$ and $U^{*}$ respectively. Also, suppose there is at least one point of $M$ such that the local tensors $W_{A}$ defined at this point are linearly independent. Then, there exists a connection on $M$ such that on each coordinate neighborhood $U, \nabla_{k} W_{A}=F_{A k}^{B} W_{B}$ for some set of local covariant vectors $F_{A k}^{B}$ if and only if for each coordinate neighborhood $U$ there is a set of linearly independent constants $C_{A}(U)$ such that for each $x \in U$ there is a frame $z(x)$ at $x$ so that $\tilde{W}_{A}(z(x))=\phi_{A}^{B}(x, U) C_{B}(U)$, where $\operatorname{det} \phi_{A}^{B}(x, U) \neq 0$, and such that if $x \in U \cap U^{*}$ and $\tilde{W}_{A}(z(x))=\phi_{A}^{B}(x, U) C_{B}(U)$, then also $\tilde{W}_{A}^{*}(z(x))=\phi_{A}^{B}\left(x, U^{*}\right) C_{B}\left(U^{*}\right)$ (i.e. the same frame is used for the $W_{A}^{*}$ as for the $\left.W_{A}\right)$.

Proof. Suppose that the $W_{A}$ 's are linearly independent at one point of $U$. Then, if there exists a connection on $M$ such that $\nabla_{k} W_{A}=F_{A k}^{B} W_{B}$ on $U$, the $W_{A}$ 's form a perfect set of tensors on $U$ and hence, by Corollary 2.3, they are linearly independent at every point of $U$. If $U \cap U^{*}$ is non-empty, we see that $\phi_{A}^{B} \phi_{B}^{C}=\delta_{A}^{C}$, where $W_{A}^{*}=\phi_{A}^{B} W_{B}$ and $W_{A}=\phi_{A}^{B} W_{B}^{*}$ on $U \cap U^{*}$, so that the $W_{A}^{*}$ are linearly independent on $U \cap U^{*}$ and, by the above reasoning, hence on all of $U^{*}$. Since $M$ is connected, we see that the local tensors 
defined for each coordinate neighborhood are linearly independent in these neighborhoods. Theorem 2.8 then proves the "only if" part of the theorem, noting that we pick only one frame in $\pi^{-1}(x) \cap B\left[z_{0}\right]$ for each $x$ in $U \cap U^{*}$.

We now prove the "if part". Take any coordinate neighborhood $U$. Define $H_{U}$ by $H_{U}=\left\{g \in G \mid g^{-1} C_{A}(U)=\eta_{A}^{B}(g) C_{B}(U)\right.$ for some $\eta_{A}^{B}(g)$ with det $\left.\eta_{A}^{B}(g) \neq 0\right\}$, where $\tilde{W}_{A}(z(x))=\phi_{A}^{B}(x, U) C_{B}(U)$ for some frame $z(x)$ at $x$. If $x \in U \cap U^{*}$, we have $\tilde{W}_{A}^{*}(z(x))=\phi_{A}^{B}\left(x, U^{*}\right) C_{B}\left(U^{*}\right)$ and

$$
\tilde{W}_{A}^{*}(z(x))=\tilde{\phi}_{A}^{B}(z(x)) \tilde{W}_{B}(z(x))=\tilde{\phi}_{A}^{B}(z(x)) \phi_{B}^{C}(x, U) C_{C}(U) \text {. }
$$

Therefore, $C_{A}\left(U^{*}\right)=\phi_{A}^{-1 D}\left(x, U^{*}\right) \tilde{\phi}_{D}^{B}(z(x)) \phi_{B}^{C}(x, U) C_{C}(U)$, and since $\operatorname{det}\left(\phi^{-1 D}\right.$ $\left.\left(x, U^{*}\right) \tilde{\phi}_{D}^{B}(z(x)) \phi_{B}^{C}(x, U)\right) \neq 0$ it is easy to see that $H_{U}=H_{U *}$. We will denote this Lie subgroup of $G$ by $H$.

Define $B_{H}(U)$ to be the set of all frames $z$ in $U$ for which $\tilde{W}_{A}(z)=$ $\xi_{A}^{B}(z) C_{B}(U)$ for some non-singular matrix $\xi_{A}^{B}(z)$. It is now easy to show that if $x \in U \cap U^{*}$, then

$$
B_{H}(U) \cap \pi^{-1}(x)=B_{H}\left(U^{*}\right) \cap \pi^{-1}(x) .
$$

Let $B_{H}$ denote the union of all the $B_{H}(U)$. In the same fashion as in the proof of Theorem 2.8, it can now be show that $B_{H}(M, H)$ is a principal fiber bundle. Therefore, if we put a connection on $B_{H}(M, H)$, it can be extended to a connection on $B(M, G)$ in such a fashion that $B_{H}\left[z_{0}\right]=B\left[z_{0}\right]$. Thus, $B\left[z_{0}\right] \cap \pi^{-1}(U) \subseteq B_{H}(U)$ for each $U$, and since the $W_{A}$ 's satisfy the hypothesis of Theorem 2.4 on $B_{H}(U)$, and therefore on $B\left[z_{0}\right] \cap \pi^{-1}(U)$, we have the $W_{A}$ 's form a perfect set of tensors on $U$. This finishes the proof of the theorem.

To prove Theorem 5.1, we note that on each coordinate neighborhood $U$, we can find a set of vectors $W_{A}^{i}$ which are a basis for the local field of $r$-planes and the global field of $r$-planes is a parallel field if and only if $\nabla_{k} W_{A}^{i}=F_{A k}^{B} W_{B}^{i}$ (see Walker [5]). Now, since the $W_{A}^{i}$ 's are linearly independent, they can be extended to a basis $W_{\alpha}^{i}(x)$ for $M_{x}$ at each $x \in U$. Denote the dual basis of $W_{a}^{i}(x)$ by $W_{i}^{\alpha}(x)$. Then, we see that

$$
\tilde{W}_{A}^{\alpha}(z(x))=W_{A}^{i}(x) W_{i}^{\alpha}(x)=\delta_{A}^{\alpha}=\delta_{A}^{B} \delta_{B}^{\alpha},
$$

where $z(x)=\left(x, W_{\alpha}^{i}(x)\right)$. If $x \in U \cap U^{*}$ and $W_{A}^{* i}(x)=\phi_{A}^{B}(x) W_{B}^{i}(x)$, then

$$
\tilde{W}_{A}^{\alpha}(z(x))=\phi_{A}^{B}(x) W_{B}^{i}(x) W_{i}^{\alpha}(x)=\phi_{A}^{B}(x) \delta_{B}^{\alpha} .
$$

Thus, it is easy to see that the hypotheses of Theorem 5.2 are satisfied and there exists a connection on $M$, with respect to which the field of planes is a parallel field of planes.

It should be noted that a generalization of Theorem 5.1 to a complete system of fields of planes can be proved directly from a special case of Theorem 
2.8. This is accomplished by using the projection tensors associated with the complete system (see Wong [8]). The generalization of Theorem 2.8 to Theorem 5.2 in order to prove Theorem 5.1 was used here because the proof then dealt directly with the local bases of the field of planes.

\section{Bibliography}

[1] S. S. Chern, Differentiable manifolds, Mimeographed notes, University of Chicago, 1959.

[2] V. Hlavaty, Holonomy group II. The Lie group induced by a tensor, J. Math. Mech. 8 (1959) 597-622.

[ 3] H. Hochstadt, Differential equations, Holt, Rinehart and Winston, New York, 1964.

[4] G. Ludden, Tensor spaces associated with a given tensor, to appear.

[5] A. G. Walker, On parallel fields of partially null vector spaces, Quart. J. Math. 20 (1949) $135-145$.

[6] T. J. Willmore, Parallel distribution on manifolds, Proc. London Math. Soc. (3) 6 (1956) 191-204.

[7] Y. C. Wong, Recurrent tensors on a linearly connected differentiable manifold, Trans. Amer. Math. Soc. 99 (1961) 325-341.

[8] Existence of linear connections with respect to which given tensor fields are parallel or recurrent, Nagoya Math. J. 24 (1964) 67-108.

Michigan State University 
